\title{
SAGES masters program: determining the seminal articles for each pathway
}

\author{
Dimitrios Stefanidis $^{1}$ (D - Linda Schultz ${ }^{2} \cdot$ Shauna Bostian $^{2} \cdot$ Patricia Sylla $^{3} \cdot$ Eric M. Pauli $^{4} \cdot$ Dmitry Oleynikov $^{5}$. \\ Marina Kurian $^{6} \cdot$ Leena Khaitan $^{7} \cdot$ Michael W. Cripps $^{8} \cdot$ Sharon Bachman $^{9} \cdot$ Adnan Alseidi $^{10} \cdot$ L. Michael Brunt $^{11}$. \\ Horacio Asbun ${ }^{12}$. Daniel B. Jones ${ }^{13}$
}

Received: 4 December 2019 / Accepted: 22 January 2020 / Published online: 12 February 2020

(c) Society of American Gastrointestinal and Endoscopic Surgeons 2020

\begin{abstract}
Background The Society of American Gastrointestinal and Endoscopic Surgeons (SAGES) has recently developed and announced its Masters Program that aims to address existing needs of practicing surgeons for lifelong learning and consists of eight clinical pathways each containing three anchoring procedures. The objective of this study was to select the seminal articles for each anchoring procedure of these pathways using a systematic methodology.

Methods A systematic literature search of Web of Science was conducted for the most cited articles for each of the anchoring procedures of the SAGES Masters pathways. The most relevant identified articles were then reviewed by expert members of the relevant SAGES pathway committees and task forces and the seminal articles chosen for each anchoring procedure using expert consensus.

Results 578 highly cited articles were identified by the original search of the literature and the seminal articles were selected for each anchoring procedure after expert review and consensus. Articles address procedural outcomes, disease pathophysiology, and surgical technique and are presented in this paper.

Conclusions We have identified seminal articles for each anchoring procedure of the SAGES Masters program pathways using a systematic methodology. These articles provide surgeon participants of this program with a great resource to improve their procedure-specific knowledge and may further benefit the larger surgical community by focusing its attention to must-read impactful work that may inform best practices.
\end{abstract}

Keywords Anchoring $\cdot$ Seminal $\cdot$ Surgery $\cdot$ Masters $\cdot$ Pathways

Dimitrios Stefanidis

dimstefa@iu.edu

1 Department of Surgery, Indiana University School of Medicine, 545 Barnhill Drive, Emerson Hall 125, Indianapolis, IN 46202, USA

2 Society of American Gastrointestinal and Endoscopic Surgeons, Los Angeles, CA, USA

3 Department of Surgery, Mount Sinai Hospital, New York, NY, USA

4 Department of Surgery, Penn State Hershey Medical Center, Hershey, PA, USA

5 Department of Surgery, University of Nebraska Medical Center, Omaha, NE, USA

6 Department of Surgery, New York University Langone Medical Center, New York, NY, USA
7 Department of Surgery, University Hospitals of Cleveland, Chardon, OH, USA

8 Department of Surgery, The University of Texas Southwestern Medical Center At Dallas, Dallas, TX, USA

9 Department of Surgery, Inova Fairfax Hospital, Falls Church, VA, USA

10 Department of Surgery, Virginia Mason, Seattle, WA, USA

11 Department of Surgery, Washington University School of Medicine, St Louis, MO, USA

12 Miami Cancer Institute, Baptist Health South Florida, Miami, FL, USA

13 Department of Surgery, Beth Israel Deaconess Medical Center, Boston, MA, USA 
The Society of American Gastrointestinal and Endoscopic Surgeons (SAGES) has recently developed and published its Masters Program [1] that aims to address existing needs of practicing surgeons for lifelong learning after training completion and serve as an optimal way for general surgeons to achieve continuous certification. The SAGES Masters Program organizes educational content across 8 clinical pathways relevant to its membership (Acute Care, Bariatric, Biliary, Colon, Foregut, Hernia and Robotic Surgery, and Flexible Endoscopy) and builds on the Dreyfus model of skill acquisition [2] by offering curricula addressing three levels of performance (competency, proficiency, and mastery). The content of each pathway and level has been determined based on expert consensus of the SAGES board and includes elements addressing knowledge, technical, and nontechnical surgical skills [1]. Each pathway level incorporates an anchoring procedure that is meant to be used for training and assessment of surgeons and coaching by more experienced members of the society. The steering group of the Masters program felt it was important that knowledge acquisition for each anchoring procedure of the 8 pathways should incorporate the best available evidence in the literature. The steering group therefore assigned experts to identify up to 10 seminal articles for each anchoring procedure using a systematic methodology.

The objective of this paper is to report the seminal articles for each anchoring procedure of the eight SAGES Masters pathways and the methodology used to determine them.

\section{Materials and methods}

To determine the seminal papers for each anchoring procedure of the 8 pathways, a systematic literature review was performed by the SAGES librarian in April 2018 for each anchoring procedure. For each identified paper, the year of publication was recorded and the Web of Science and Google Scholar were then searched to assess the number of citations received since publication. To assess the impact of each paper in the field also taking into account the duration since publication, a citation index (CI) was calculated using the equation: $\mathrm{CI}=$ number of citations/ years since publication. All articles were then ranked based on this CI and the top 30 papers with the highest $\mathrm{CI}$ were selected. These 30 articles were then reviewed by members of the SAGES committees or task forces relevant to each pathway. Reviewing members had expertise in the respective pathways and procedures; the leaders of each relevant SAGES committee/ task force were also encouraged to obtain input from other well-known experts in the field as necessary. To select the top papers, experts were encouraged to select articles that they felt should be read by every surgeon performing the relevant procedure and that had known impact in the field taking into consideration the results of the literature search and CI for each paper. The expert group was allowed to include manuscripts not identified by the literature search if they were deemed to be important by consensus. The final seminal papers for each procedure of each pathway were selected based on expert consensus and are reported here; up to 10 articles per procedure were requested. Of note, the robotic pathway is an adjunct pathway without specific anchoring procedures as the technique applies to multiple procedures; hence seminal articles relevant to the robotic technique are listed together rather than according to specific procedures.

This study was exempt from IRB approval.

\section{Results}

160 SAGES Committee/ Task Force members and other experts participated in the sentinel article selection process. 578 articles for all procedures were retrieved initially by our search of which up to 30 per procedure were subjected to the expert review and consensus process. The $\mathrm{CI}$ for the seminal articles across all pathways for Google Scholar ranged from 0.25 to 257.2 and for Web of Science 0.25-140.44. Articles addressed procedural outcomes, disease pathophysiology, and surgical technique. Selected articles were published in a variety of surgical journals and originated from several different countries. The seminal articles for each procedure are reported on Tables 1, 2, 3, 4, 5, 6, 7, 8 . 
Table 1 Seminal articles for acute surgery pathway

1a: Laparoscopic appendectomy

Article Reference

CI

Google Scholar

Web of Science

Aziz O, Athanasiou T, Tekkis PP, Purkayastha S, Haddow J, Malinovski V, Paraskeva P, Darzi A (2006) Laparoscopic versus 26.8 open appendectomy in children: a meta-analysis. Ann Surg 243:17-27

Chung RS, Rowland DY, Li P, Diaz J (1999) A meta-analysis of randomized controlled trials of laparoscopic versus conven- 20.7 tional appendectomy. Am J Surg 177:250-256

9.5

Yau KK, Siu WT, Tang CN, Yang GP, Li MK (2007) Laparoscopic versus open appendectomy for complicated appendicitis. J 21.8 Am Coll Surg 205:60-65

Guller U, Hervey S, Purves H, Muhlbaier LH, Peterson ED, Eubanks S, Pietrobon R (2004) Laparoscopic versus open appendectomy: outcomes comparison based on a large administrative database. Ann Surg 239:43-52

Katkhouda N, Mason RJ, Towfigh S, Gevorgyan A, Essani R (2005) Laparoscopic versus open appendectomy: a prospective randomized double-blind study. Ann Surg 242: 439-448

\subsection{2}

39.2

20.28

35.5

$\mathbf{1 5 . 1 5}$

Li X, Zhang J, Sang L, Zhang W, Chu Z, Li X, Liu Y (2010) Laparoscopic versus conventional appendectomy-a meta-analysis 34.9 of randomized controlled trials. BMC Gastroenterol 10:129

Temple LK, Litwin DE, McLeod RS (1999) A meta-analysis of laparoscopic versus open appendectomy in patients suspected of having acute appendicitis. Can J Surg 42:377-383

\section{6}

11.8

4.38

Frazee RC, Roberts JW, Symmonds RE, Snyder SK, Hendricks JC, Smith RW, Custer MD 3rd, Harrison JB (1994) A prospec- 13.6 tive randomized trial comparing open versus laparoscopic appendectomy. Ann Surg 219:725-728

7.16

19.5

Ortega AE, Hunter JG, Peters JH, Swanstrom LL, Schirmer B (1995) A prospective, randomized comparison of laparoscopic appendectomy with open appendectomy. Laparoscopic Appendectomy Study Group. Am J Surg 169:208-212

Attwood SE, Hill AD, Murphy PG, Thornton J, Stephens RB (1992) A prospective randomized trial of laparoscopic versus open appendectomy. Surgery 112:497-501

1b: Laparoscopic cholecystectomy for acute cholecystitis

Article Reference

15.6

9.88

CI

Google Scholar

Web of Science

Rattner DW, Ferguson C, Warshaw AL (1993) Factors associated with successful laparoscopic cholecystectomy for acute cholecystitis. Ann Surg 217:233-236

11.8

7.32

Lau H, Lo CY, Patil NG, Yuen WK (2006) Early versus delayed-interval laparoscopic cholecystectomy for acute cholecystitis: 23.6 a meta-analysis. Surg Endosc 20:82-87

12.30

Banz V, Gsponer T, Candinas D, Güller U (2011) Population-based analysis of 4113 patients with acute cholecystitis: defining 23.0

the optimal time-point for laparoscopic cholecystectomy. Ann Surg 254:964-970

10.5

Gurusamy KS, Samraj K (2006) Early versus delayed laparoscopic cholecystectomy for acute cholecystitis. Cochrane Database 22.1

Syst Rev 18:CD005440

Gurusamy K, Samraj K, Gluud C, Wilson E, Davidson BR (2010) Meta-analysis of randomized controlled trials on the safety and effectiveness of early versus delayed laparoscopic cholecystectomy for acute cholecystitis. Br J Surg 97:141-150

3.25

42.8

19.87

Borzellino G, Sauerland S, Minicozzi AM, Verlato G, Di Pietrantonj C, de Manzoni G, Cordiano C (2008) Laparoscopic chol- 18.6

ecystectomy for severe acute cholecystitis. A meta-analysis of results. Surg Endosc 22:8-15

8.7

Chandler CF, Lane JS, Ferguson P, Thompson JE, Ashley SW (2000) Prospective evaluation of early versus delayed laparoscopic cholecystectomy for treatment of acute cholecystitis. Am Surg 66:896-900

13.1

6.77

Siddiqui T, MacDonald A, Chong PS, Jenkins JT (2008) Early versus delayed laparoscopic cholecystectomy for acute cholecystitis: a meta-analysis of randomized clinical trials. Am J Surg 195:40-47

30.7

12.6

19.6

9.42

ecystectomy for acute cholecystitis: a prospective randomized trial. Surg Endosc 18:1323-1327

27.9

15.1

tectomy for acute cholecystitis. Ann Surg 227:461-467

1c: Laparoscopic management of GI perforation

Article Reference

CI

Google Scholar

Web of Science

Bertleff MJ, Halm JA, Bemelman WA, van der Ham AC, van der Harst E, Oei HI, Smulders JF, Steyerberg EW, Lange JF 20.4

(2009) Randomized clinical trial of laparoscopic versus open repair of the perforated peptic ulcer: the LAMA Trial. World J 7.11

Surg 33:1368-1373 
Table 1 (continued)

1c: Laparoscopic management of GI perforation

Article Reference

CI

Google Scholar

Web of Science

Livingston EH, Woodward WA, Sarosi GA, Haley RW (2007) Disconnect between incidence of nonperforated and perforated appendicitis: implications for pathophysiology and management. Ann Surg 245:886-892

27.5

13.63

Lüning TH, Keemers-Gels ME, Barendregt WB, Tan AC, Rosman C (2007) Colonoscopic perforations: a review of 30,366 patients. Surg Endosc 21:994-997

Siu WT, Leong HT, Law BK, Chau CH, Li AC, Fung KH, Tai YP, Li MK (2002) Laparoscopic repair for perforated peptic ulcer: a randomized controlled trial. Ann Surg 235:313-319

Myers E, Hurley M, O’Sullivan GC, Kavanagh D, Wilson I, Winter DC (2008) Laparoscopic peritoneal lavage for generalized peritonitis due to perforated diverticulitis. Br J Surg 95:97-101

Bertleff MJ, Lange JF (2010) Laparoscopic correction of perforated peptic ulcer: first choice? A review of literature. Surg Endosc 24:1231-1239

Lau H (2004) Laparoscopic repair of perforated peptic ulcer: a meta-analysis. Surg Endosc 18:1013-1021

14.27

22.4

9.5

35.3

20.6

24.4

8.62

13.9

5.21

15.4

8.5

RJ, Boom MJ, Kruyt PM, Swank DJ, Steup WH, de Graaf EJ, Weidema WF, Pierik RE, Prins HA, Stockmann HB, Tollenaar RA, van Wagensveld BA, Coene PP, Slooter GD, Consten EC, van Dujin EB, Gerhards MF, Hoofwijk AG, Karsten T, Neijenhuis PA, Blanken-Peeters CF, Cense HA, Mannaerts GH, Bruin SC, Eijsbouts QA, Wiezer MJ, Hazebroek EJ, van Geloven AA, Maring JK, D'Hoore AJ, Kartheuser A, Remue C, van Grevenstein HM, Konsten JL, van der Peet DL, Govaert MJ, Engel AF, Reitsma JB, Bemelman WA, Dutch Diverticular Disease (3D) Collaborative Study Group (2010) The ladies trial: laparoscopic peritoneal lavage or resection for purulent peritonitis and Hartmann's procedure or resection with primary anastomosis for purulent or fecal peritonitis in perforated diverticulitis (NTR2037). BMC Surg 10:29

Lau WY, Leung KL, Kwong KH, Davey IC, Robertson C, Dawson JJ, Chung SC, Li AK (1996) A randomized study comparing laparoscopic versus open repair of perforated peptic ulcer using suture or sutureless technique. Ann Surg 224:131-138

Lunevicius R, Morkevicius M (2005) Systematic review comparing laparoscopic and open repair for perforated peptic ulcer. Br J Surg 92:1195-1207

Table 2 Seminal articles for bariatric surgery pathway

2a: Gastric banding procedures

Article Reference

CI Google

Scholar

Web of Science

Nguyen NT, Slone JA, Nguyen XM, Hartman JS, Hoyt DB (2009) A prospective randomized trial of laparoscopic gastric bypass versus laparoscopic adjustable gastric banding for the treatment of morbid obesity: outcomes, quality of life, and costs. Ann Surg 250:631-641

Choi J, Digiorgi M, Milone L, Schrope B, Olivera-Rivera L, Daud A, Davis D, Bessler M (2010) Outcomes of laparoscopic adjustable gastric banding in patients with low body mass index. Surg Obes Relat Dis 6:367-371

Lazzati A, De Antonio M, Paolino L, Martini F, Azoulay D, Iannelli A, Katsahian S (2017) Natural History of Adjustable Gastric Banding: Lifespan and Revisional Rate: A Nationwide Study on Administrative Data on 53,000 Patients. Ann Surg 265:439-445

Nguyen NT, Hohmann S, Nguyen XM, Elliott C, Masoomi H (2012) Outcome of laparoscopic adjustable gastric banding and prevalence of band revision and explantation at academic centers: 2007-2009. Surg Obes Relat Dis 8:724-727

O’Brien PE, MacDonald L, Anderson M, Brennan L, Brown WA (2013) Long-term outcomes after bariatric surgery: fifteenyear follow-up of adjustable gastric banding and a systematic review of the bariatric surgical literature. Ann Surg 257:87-94

Sudan R, Maciejewski ML, Wilk AR, Nguyen NT, Ponce J, Morton JM (2017) Comparative effectiveness of primary bariatric operations in the United States. Surg Obes Relat Dis 13:826-834

Schmitt F, Riquin E, Beaumesnil M, Dinomais M, Topart P, Weil D, Malka J, Coutant R, Podevin G, Bouhours-Nouet N Surg 51:403-408 
Table 2 (continued)

2a: Gastric banding procedures

Article Reference

CI Google

Scholar

Web of Science

Gulkarov I, Wetterau M, Ren CJ, Fielding GA (2008) Hiatal hernia repair at the initial laparoscopic adjustable gastric band operation reduces the need for reoperation. Surg Endosc 22:1035-1041

Chevallier JM, Paita M, Rodde-Dunet MH, Marty M, Nogues F, Slim K, Basdevant A (2007) Predictive factors of outcome after gastric banding: a nationwide survey on the role of center activity and patients' behavior. Ann Surg 246:1034-1039

Spaniolas K, Bates AT, Docimo S Jr, Obeid NR, Talamini MA, Pryor AD (2017) Single-stage conversion from adjustable gastric banding to sleeve gastrectomy or Roux-en-Y gastric bypass: an analysis of 4875 patients. Surg Obes Relat Dis

1.0 13:1880-1884

2b: Laparoscopic sleeve gastrectomy

Article Reference

CI

Google Scholar

Web of Science

Berger ER, Clements RH, Morton JM, Huffman KM, Wolfe BM, Nguyen NT, Ko CY, Hutter MM (2016) The Impact of Differ- 20.5 ent Surgical Techniques on Outcomes in Laparoscopic Sleeve Gastrectomies: The First Report from the Metabolic and Bariatric 11 Surgery Accreditation and Quality Improvement Program (MBSAQIP). Ann Surg 264:464-473

Rosenthal, RJ, International Sleeve Gastrectomy Expert Panel, Diaz AA, Arvidsson D, Baker RS, Basso N, Bellanger D, Boza 72.7 C, El Mourad H, France M, Gagner M, Galvao-Neto M, Higa KD, Himpens J, Hutchinson CM, Jacobs M, Jorgensen JO, Jossart 56.83 G, Lakdawala M, Nguyen NT, Nocca D, Prager G, Pomp A, Ramos AC, Rosenthal RJ, Shah S, Vix M, Wittgrove A, Zundel N (2012) International Sleeve Gastrectomy Expert Panel Consensus Statement: best practice guidelines based on experience of $>12,000$ cases. Surg Obes Relat Dis 8:8-19

Karamanakos SN, Vagenas K, Kalfarentzos F, Alexandrides TK (2008) Weight loss, appetite suppression, and changes in 74.1 fasting and postprandial ghrelin and peptide YY levels after Roux-en-Y gastric bypass and sleeve gastrectomy: a prospective, double-blind study. Ann Surg 247:401-407

Himpens J, Dobbeleir J, Peeters G (2010) Long-term results of laparoscopic sleeve gastrectomy for obesity. Ann Surg 252:319- 70.3 324

Hutter MM, Schirmer BD, Jones DB, Ko CY, Cohen ME, Merkow RP, Nguyen, NT (2011) First report from the American Col- 61.1 lege of Surgeons Bariatric Surgery Center Network: laparoscopic sleeve gastrectomy has morbidity and effectiveness positioned $\mathbf{3 8 . 4 2}$ between the band and the bypass. Ann Surg 254:410-420

Himpens J, Dapri G, Cadière, GB (2006) A prospective randomized study between laparoscopic gastric banding and laparoscopic isolated sleeve gastrectomy: results after 1 and 3 years. Obes Surg 16:1450-1456

Weiner RA, Weiner S, Pomhoff I, Jacobi C, Makarewicz W, Weigand, G Laparoscopic sleeve gastrectomy-influence of sleeve 33.6 size and resected gastric volume. Obes Surg 17:1297-1305

Nocca D, Krawczykowsky D, Bomans B, Noël P, Picot MC, Blanc PM, de Seguin de Hons C, Millat B, Gagner M, Monnier L, 28.1 Fabre JM (2008) A prospective multicenter study of 163 sleeve gastrectomies: results at 1 and 2 years. Obes Surg 18:560-565 19.5 Gehrer S, Kern B, Peters T, Christoffel-Courtin C, Peterli R (2010) Fewer nutrient deficiencies after laparoscopic sleeve gastrec- 33.1 tomy (LSG) than after laparoscopic Roux-Y-gastric bypass (LRYGB)-a prospective study. Obes Surg 20:447-453

Gagner M, Hutchinson C, Rosenthal R (2016) Fifth International Consensus Conference: current status of sleeve gastrectomy. 21.0 Surg Obes Relat Dis 12:750-756

2c: Laparoscopic Roux-en-Y gastric bypass

Article Reference

CI

Google Scholar

Web of Science

Schauer PR, Bhatt DL, Kirwan JP, Wolski K, Aminian A, Brethauer SA, Navaneethan SD, Singh RP, Pothier CE, Nissen SE, Kashyap SR, STAMPEDE investigators (2017) Bariatric Surgery versus Intensive Medical Therapy for Diabetes- 5 Year Outcomes N Engl J Med 376: 641-651

Buchwald H, Estok R, Fahrbach K, Banel D, Jensen MD, Pories WJ, Bantle JP, Sledge I (2009) Weight and type 2 diabetes after bariatric surgery: systematic review and meta-analysis. Am J Med 122: 248-256.e5

Nguyen NT, Goldman C, Rosenquist CJ, Arango A, Cole CJ, Lee SJ, Wolfe BM (2001) Laparoscopic versus open gastric bypass: a randomized study of outcomes, quality of life, and costs. Ann Surg 234:279-291 
Table 2 (continued)

2c: Laparoscopic Roux-en-Y gastric bypass

Article Reference

CI

Google Scholar

Web of Science

Longitudinal Assessment of Bariatric Surgery (LABS) Consortium, Flum, DR, Belle SH, King WC, Wahed AS, Berk P,

120.1

Chapman W, Pories W, Courcoulas A, McCloskey C, Mitchell, J, Patterson E, Pomp A, Staten MA, Yanovski SZ, Thirlby R, 77.33

Wolfe B (2009) Perioperative safety in the longitudinal assessment of bariatric surgery. N Engl J Med 361:445-454

Rubino F, Gagner M, Gentileschi P, Kini S, Fukuyama S, Feng J, Diamond E (2004) The early effect of the Roux-en-Y gastric 47.6

bypass on hormones involved in body weight regulation and glucose metabolism. Ann Surg 240:236-242

23.12

Buchwald H, Estok R, Fahrbach K, Banel D, Sledge I (2007) Trends in mortality in bariatric surgery: a systematic review and 61.7 meta-analysis. Surg 142: 621-32

32.63

Maggard MA, Shugarman LR, Suttorp M, Maglione M, Sugerman HJ, Livingston EH, Nguyen NT, Li Z, Mojica WA, Hilton 125.3

L, Rhodes S, Morton SC, Shekelle PG (2005) Meta-analysis: surgical treatment of obesity. Ann Int Med 142:547-559

66.84

Adams TD, Gress RE, Smith SC, Halverson RC, Simper SC, Rosamond WD, Lamonte MJ, Stroup AM, Hunt SC (2007)

Long-term mortality after gastric bypass surgery. NEJM 357:753-761

119.81

Higa KD, Boone KB, Ho T (2000) Complications of the laparoscopic Roux-en-Y gastric bypass: 1,040 patients-what have we 35.5

learned? Obes Surg 10:509-513

21.22

Comeau E, Gagner M, Inabnet WB, Herron DM, Quinn T, Pomp A (2005) Symptomatic internal hernias after laparoscopic bariatric surgery. Surg Endosc 19:34-39

8.5

5.46

2d: Laparoscopic bariatric redo procedures

Article Reference

Shimizu H, Annaberdyev S, Motamarry I, Kroh M, Schauer PR, Brethauer SA (2013) Revisional bariatric surgery for unsuccessful weight loss and complications. Obes Surg 23:1766-1773

Podnos YD, Jimenez JC, Wilson SE, Stevens CM, Nguyen NT (2003) Complications after laparoscopic gastric bypass: a review of 3464 cases. Arch Surg 138:957-961

51

DeMaria EJ, Sugerman HJ, Meador JG, Doty JM, Kellum JM, Wolfe L, Szucs RA, Turner MA (2001) High failure rate after

Paroz A, Calmes JM, Giusti V, Suter M (2006) Internal hernia after laparoscopic Roux-en-Y gastric bypass for morbid obesity: 11.5 a continuous challenge in bariatric surgery. Obes Surg 16:1482-1487

Brolin RE, Cody RP (2008) Weight loss outcome of revisional bariatric operations varies according to the primary procedure. $\quad 7.4$ Ann Surg 248:227-232

Patel S, Szomstein S, Rosenthal RJ (2011) Reasons and outcomes of reoperative bariatric surgery for failed and complicated procedures (excluding adjustable gastric banding). Obes Surg 21:1209-1219

Gagner M, Gentileschi P, de Csepel J, Kini S, Patterson E, Inabnet WB, Herron D, Pomp A (2002) Laparoscopic reoperative bariatric surgery: experience from 27 consecutive patients. Obes Surg 12:254-260

Horgan S, Jacobsen G, Weiss GD, Oldham JS Jr, Denk PM, Borao F, Gorcey S, Watkins B, Mobley J, Thompson K, Spivack A, Voellinger D, Thompson C, Swanstrom L, Shah P, Haber G, Brengman M, Schroder G (2010) Incisionless revision of post-Roux-en-Y bypass stomal and pouch dilation: multicenter registry results. Surg Obes Relat Dis 6:290-295

Ardestani A, Lautz DB, Tavakkolizadeh A (2011) Band revision versus Roux-en-Y gastric bypass conversion as salvage opera- 8.0 tion after laparoscopic adjustable gastric banding. Surg Obes Relat Dis 7:33-37

Gagner M, Rogula T (2003) Laparoscopic reoperative sleeve gastrectomy for poor weight loss after biliopancreatic diversion with duodenal switch. Obes Surg 13:649-654 
Table 3 Seminal articles for biliary pathway

3a: Laparoscopic cholecystectomy

Article Reference

CI

Google Scholar

Web of Science

Duncan CB, Riall TS (2012) Evidence-based current surgical practice: calculous gallbladder disease. J Gastrointest Surg 16: 2011-2025

Pucher PH, Brunt LM, Fanelli RD, Asbun HJ, Aggarwal R (2015) SAGES expert Delphi consensus: critical factors for safe surgical practice in laparoscopic cholecystectomy. Surg Endosc 29:3074-3085

Way LW, Stewart L, Gantert W, Liu K, Lee CM, Whang K, Hunter JG (2003) Causes and prevention of laparoscopic bile duct 45.7 injuries. Analysis of 252 cases from a human factors and cognitive psychology perspective. Ann Surg 237:460-469

21.1

Strasberg SM, Hertl M, Soper NJ (1995) An analysis of the problem of biliary injury during laparoscopic cholecystectomy. J 68.4

Am Coll Surg 180:101-125

Strasberg SM (2008) Error traps and vasculo-biliary injury in laparoscopic and open cholecystectomy. J Hepatobiliary Pancreat Surg 15:284-292

Strasberg SM, Brunt LM (2010) Rationale and use of the critical view of safety in laparoscopic cholecystectomy. J Am Coll Surg 211:132-138

Davidoff AM, Pappas TN, Murray EA, Hilleren DJ, Johnson RD, Baker ME, Newman GE, Cotton PB, Meyers WC (1992)

Mechanisms of major biliary injury during laparoscopic cholecystectomy. Ann Surg. 215(3):196-202

Sanford DE, Strasberg SM (2014) A simple effective method for generation of a permanent record of the Critical View of

Safety during laparoscopic cholecystectomy by intraoperative "doublet" photography. J Am Coll Surg. 218:170-178

Nijssen MA, Schreinemakers JM, Meyer Z, van der Schelling GP, Crolla RM, Rijken AM (2015) Complications after laparoscopic cholecystectomy: A video evaluation study of whether the critical view of safety was reached. World J Surg 39:17981803

Lo CM, Liu CL, Fan ST, Lai EC, Wong J (1998) Prospective randomized study of early versus delayed laparoscopic cholecystectomy for acute cholecystitis. Ann Surg. 227(4):461-7

Nijssen MA, Schreinemakers JM, van der Schelling GP, Crolla, RM, Rijken AM (2016) Improving critical view of safety in laparoscopic cholecystectomy by teaching interventions. J Surg Educ 73:442-447

3b: Intraoperative cholangiogram

Article Reference

\section{CI}

Google Scholar

Web of Science

Ludwig K, Bernhardt J, Steffen H, Lorenz D (2002) Contribution of intraoperative cholangiography to incidence and outcome of common bile duct injuries during laparoscopic cholecystectomy. Surg Endosc 16:1098-1104

Flum DR, Dellinger EP, Cheadle A, Chan L, Koepsell T (2003) Intraoperative cholangiography and risk of common bile duct injury during cholecystectomy. JAMA 289:1639-1644

Törnqvist B, Strömberg C, Persson G, Nilsson M (2012) Effect of intended intraoperative cholangiography and early detection of bile duct injury on survival after cholecystectomy: population-based cohort study. BMJ 345:e6457

Ford JA, Soop M, Du J, Loveday BPT, Rodgers M (2012) Systematic review of intraoperative cholangiography in cholecystectomy. Br J Surg 99:160-167

Buddingh KT, Nieuwenhuijs VB, van Buuren L, Hulscher JB, de Jong JS, van Dam GM (2012) Intraoperative assessment of biliary anatomy for prevention of bile duct injury: A review of current and future patient safety interventions. Surg Endosc 25:2449-2461

Vlek SL, van Dam DA, Rubinstein SM, de Lange-de Klerk ESM, Schoonmade LJ, Tuynman JB, Meijerink WJHJ, Ankersmit M (2017) Biliary tract visualization using near-infrared imaging with indocyanine green during laparoscopic cholecystectomy: results of a systematic review. Surg Endosc 31:2731-2742

Falcone RA Jr, Fegelman EJ, Nussbaum MS, Brown DL, Bebbe TM, Merhar GL, Johannigman JA, Luchette FA, Davis K Jr, Hurst JM (1999) A prospective comparison of laparoscopic ultrasound vs intraoperative cholangiogram during laparoscopic cholecystectomy. Surg Endosc 13(8):784-8

Yokoe M, Hata J, Takada T, Strasberg SM, Asbun H, Wakabayashi G, Kozaka K, Endo I, Deziel DJ, Miura F, Okamoto K, Hwang TL, HuangWS, Ker CG, Chen MF, Han HS, Yoon YS, Choi IS, Yoon DS, Noguchi Y, Shikata S, Ukai T, Higuchi R, Gabata T, Mori Y, Iwashita Y, Hibi T, Jagannath P, Jonas E, Liau KH, Dervenis C, Gouma DJ, Cherqui D, Belli G, Garden OJ, Giménez ME, de Santibañes E, Suzuki K, Umezawa A, Supe AN, Pitt HA, Singh H, Chan ACW, Lau WY, Teoh AYB, Honda G, Sugioka A, Asai K, Gomi H, Itoi T, Kiriyama S, Yoshida M, Mayumi T, Matsumura N, Tokumura H, Kitano S, Hirata K, Inui K, Sumiyama Y, Yamamoto M (2018) Tokyo guidelines 2018: diagnostic criteria and severity grading of acute cholecystitis (with videos). J Hepatobil Pancr Sci; 25: 41-54 
Table 3 (continued)

3b: Intraoperative cholangiogram

Article Reference

CI

Google Scholar

Web of Science

Cao AM, Eslick GD, Cox MR (2015) Early cholecystectomy is superior to delayed cholecystectomy for acute cholecystitis: a 10.8

meta-analysis. J Gastrointest Surg 19: 848-857

6.3

Varadarajulu S, Eloubeidi MA, Wilcox CM, Hawes RH, Cotton PB (2006) Do all patients with abnormal intraoperative cholangiogram merit endoscopic retrograde cholangiopancreatography? Surg Endosc 20(5):801-5

1.8

1.1

Roulin D, Saadi A, Di Mare L, Demartines N, Halkic N (2016) Early versus delayed cholecystectomy for acute cholecystitis, 16.3 are the $72 \mathrm{~h}$ Still the rule?: a randomized trial. Ann Surg 264:717-722

12.3

Wu XD, Tian X, Liu MM, Wu L, Zhao S, Zhao L (2015) Meta-analysis comparing early versus delayed laparoscopic chol- 15.8 ecystectomy for acute cholecystitis. Br J Surg 102:1302-1313

9.0

3c: Laparoscopic common bile duct exploration

Article Reference

CI

Google Scholar

Web of Science

Bansal VK, Misra MC, Rajan K, Kilambi R, Kumar S, Krishna A, Kumar A, Pandav CS, Subramaniam R, Arora MK, Garg PK (2014) Single-stage laparoscopic common bile duct exploration and cholecystectomy versus two-stage endoscopic stone extraction followed by laparoscopic cholecystectomy for patients with concomitant gallbladder stones and common bile duct stones: a randomized controlled trial. Surg Endosc 28(3):875-85

Berci G, Hunter J, Morgenstern L, Arregui M, Brunt M, Carroll B, Edye M, Fermelia D, Ferzli G, Greene F, Petelin J, Phillips 7.8

E, Ponsky J, Sax H, Schwaitzberg S, Soper N, Swanstrom L, Traverso W (2013) Laparoscopic cholecystectomy. Laparo-

scopic cholecystectomy: first, do no harm; second, take care of bile duct stones. Surg Endosc 27:1051-1054

Schwab B, Teitelbaum EN, Barsuk JH, Soper NJ, Hungness ES (2018) Single-stage laparoscopic management of choledocholithiasis: an analysis after implementation of a mastery learning resident curriculum. Surgery 163:503-508

Pan L, Chen M, Ji L, Zheng L, Yan P, Fang J, Zhang B, Cai X (2018) The safety and efficacy of laparoscopic common bile $\mathbf{5 . 0}$ duct exploration combined with cholecystectomy for the management of cholecysto-choledocholithiasis: An up to date metaanalysis. Ann Surg 268:247-253

Pang L Zhang Y, Wang Y, Kong J (2018) Transcystic versus traditional laparoscopic common bile duct exploration: its advantages and a meta-analysis. Surg Endosc 32:4363-4376

Koc B, Karahan S, Adas G, Tutal F, Guven H, Ozsoy A. (2013) Comparison of laparoscopic common bile duct exploration and endoscopic retrograde cholangiopancreatography plus laparoscopic cholecystectomy for choledocholithiasis: a prospective randomized study. Am J Surg 206(4):457-63

Somasekar K, Chan DSY, Sreekumar NS, Anwer A (2018) Choledocholithiasis after bariatric surgery - more than a stone's throw to reach? J Gastrointest Surg 22:529-537

Petelin JB (2003) Laparoscopic common bile duct exploration. Surg Endosc 17(11):1705-15

Podda M, Polignano FM, Luhmann A, Wilson MS, Kulli C, Tait IS (2016) Systematic review with meta-analysis comparing primary duct closure and T-tube drainage after laparoscopic common bile duct exploration for choledocholithiasis. Surg Endosc 30:845-861

Erben Y, Benavente-Chenhalls LA, Donohue JM, Que FG, Kendrick ML, Reid-Lombardo KM, Farrell MB, Nagorney DM (2011) Diagnosis and treatment of Mirizzi syndrome: 23-year Mayo Clinic experience. J Am Coll Surg 213:114-121

Strasberg SM, Pucci MJ, Brunt LM, Deziel DJ (2016) Subtotal Cholecystectomy-"Fenestrating" vs "Reconstituting" subtypes and the prevention of bile duct injury: definition of the optimal procedure in difficult operative conditions. J Am Coll Surg 222:89-96

van Dijk AH, Donkervoort SC, Lameris W, de Vries E, Eijsbouts QAJ, Vrouenraets BC, Busch OR, Boermeester MA, de Reuver PR (2017) Short and long-term outcomes after a reconstituting and fenestrating subtotal cholecystectomy. J Am Coll Surg 225:371-379 
Table 4 Seminal articles for colorectal pathway

4a: Laparoscopic right colectomy

Article Reference

CI

Google Scholar

Web of Sci-

ence

Dijkstra FA, Bosker RJ, Veeger NJ, van Det MJ, Pierie JP (2015) Procedural key steps in laparoscopic colorectal surgery, consensus through Delphi methodology. Surg Endosc 29:2620-2627

Liang JT, Lai HS, Lee PH (2007) Laparoscopic medial-to-lateral approach for the curative resection of right-sided colon cancer. 4.4 Ann Surg Oncol 14:1878-1879

Rickard MJFX, Keshava A, Toh JWT (2017) Three steps and a join: a simple guide to right- and left-sided medial-to-lateral laparoscopic colorectal surgery. Tech Coloproctol 21:673-677

Benlice C, Stocchi L, Costedio MM, Gorgun E, Kessler H (2016) Impact of the Specific Extraction Site Location on the Risk of 4.5 Incisional Hernia After Laparoscopic Colorectal Resection. Dis Colon Rectum 59:743-750

Lee SJ, Park SC, Kim MJ, Sohn DK, Oh JH (2016) Vascular Anatomy in Laparoscopic Colectomy for Right Colon Cancer. Dis 3.5

Colon Rectum 59:718-724

Rondelli F, Trastulli S, Avenia N, Schillaci G, Cirocchi R, Gullà N, Mariani E, Bistoni G, Noya G (2012) Is laparoscopic right 8.7 colectomy more effective than open resection? A meta-analysis of randomized and nonrandomized studies. Colorectal Dis $\mathbf{5 . 1 6}$ 14:e447-469

Cabot JC, Lee SA, Yoo J, Nasar A, Whelan RL, Feingold DL (2010) Long-term consequences of not closing the mesenteric 4.6 defect after laparoscopic right colectomy. Dis Colon Rectum 53:289-292

Adamina M, Manwaring ML, Park KJ, Delaney CP (2012) Laparoscopic complete mesocolic excision for right colon cancer. 15.2

Surg Endosc 26:2976-2980

7.0

van Oostendorp S, Elfrink A, Borstlap W, Schoonmade L, Sietses C, Meijerink J, Tuynman J (2012) Intracorporeal versus 1.8 extracorporeal anastomosis in right hemicolectomy: a systematic review and meta-analysis. Surg Endosc 31:64-77

Tekkis PP1, Senagore AJ, Delaney CP, Fazio VW (2005) Evaluation of the learning curve in laparoscopic colorectal surgery: 47.5 comparison of right-sided and left-sided resections. Ann Surg 242:83-91

4b: Laparoscopic left colectomy

Article Reference

Rickard MJFX, Keshava A, Toh JWT (2017) Three steps and a join: a simple guide to right- and left-sided medial-to-lateral laparoscopic colorectal surgery. Tech Coloproctol 21:673-677

Milone M, Milone F (2017) Segmental left colectomy: a modified caudal-to-cranial approach. Surg Endosc 31:1487

Kamal T, Pai A, Velchuru VR, Zawadzki M, Park JJ, Marecik SJ, Abcarian H, Prasad LM (2015) Should anastomotic assessment with flexible sigmoidoscopy be routine following laparoscopic restorative left colorectal resection? Colorectal Dis 5.7 17:160-164

Midura EF, Hanseman D, Davis BR, Atkinson SJ, Abbott DE, Shah SA, Paquette IM (2015) Risk factors and consequences of anastomotic leak after colectomy: a national analysis. Dis Colon Rectum 58:333-338

Masoni L, Mari FS, Nigri G, Favi F, Gasparrini M, Dall'Oglio A, Pindozzi F, Pancaldi A, Brescia A (2013) Preservation of 4.8

the inferior mesenteric artery via laparoscopic sigmoid colectomy performed for diverticular disease: real benefit or technical $\mathbf{2 . 2}$ challenge: a randomized controlled clinical trial. Surg Endosc 27:199-206

Schlussel AT, Wiseman JT, Kelly JF, Davids JS, Maykel JA, Sturrock PR, Sweeney WB, Alavi K (2017) Location is everything: The role of splenic flexure mobilization during colon resection for diverticulitis. Int J Surg 40:124-129 
Table 4 (continued)

4b: Laparoscopic left colectomy

Article Reference

CI

Google Scholar

Web of Science

Bonnet S1, Berger A, Hentati N, Abid B, Chevallier JM, Wind P, Delmas V, Douard R (2012) High tie versus low tie vascular ligation of the inferior mesenteric artery in colorectal cancer surgery: impact on the gain in colon length and implications on 6.7 the feasibility of anastomoses. Dis Colon Rectum 55:515-21

Leraas HJ, Ong CT, Sun Z, Adam MA, Kim J, Gilmore BF, Ezekian B, Nag US, Mantyh CR, Migaly J (2017) Hand-Assisted Laparoscopic Colectomy Improves Perioperative Outcomes Without Increasing Operative Time Compared to the Open Approach: a National Analysis of 8791 Patients. J Gastrointest Surg 21:684-691

Braga M, Frasson M, Zuliani W, Vignali A, Pecorelli N, Di Carlo V (2010) Randomized clinical trial of laparoscopic versus open left colonic resection. Br J Surg 97:1180--1186

Tekkis PP, Senagore AJ, Delaney CP, Fazio VW (2005) Evaluation of the learning curve in laparoscopic colorectal surgery: comparison of right-sided and left-sided resections. Ann Surg 242:83-91

4c: Laparoscopic left colectomy and splenic flexure release for complex inflammatory disease or advanced cancer

Article Reference
5.0

3.2

15.4

9.12

47.5

33.38
CI

Google Scholar Web of Sci-

ence

Dumont F, Da Re C, Goéré D, Honoré C, Elias D (2013) Options and outcome for reconstruction after extended left hemicolec- 1.2 tomy. Colorectal Dis 15:747-54

Mishra A, Gosselink MP, Mortensen NJ, George BD, Cunningham C, Lindsey I, Guy R, Jones OM, Hompes R (2015) Problem 0.25 solving after marginal artery injury during splenic flexure mobilization a video vignette. Colorectal Dis 17:174-175

Sciuto A, Grifasi C, Pirozzi F, Leon P, Pirozzi RE, Corcione F (2016) Laparoscopic Deloyers procedure for tension-free anasto- 1.0 mosis after extended left colectomy: technique and results. Tech Coloproctol 20:865-869

Dapri G, Bascombe NA, Cadière GB, Marks JH (2017) The three approaches to the colonic splenic flexure mobilization-a 2.2 video vignette. Colorectal Dis 19:948-949

Kim HJ, Kim CH, Lim SW, Huh JW, Kim YJ, Kim HR (2013) An extended medial-to-lateral approach to mobilize the splenic flexure during laparoscopic low anterior resection. Colorectal Dis 15:e93-98

Benseler V, Hornung M, Iesalnieks I, von Breitenbuch P, Glockzin G, Schlitt HJ, Agha A (2012) Different approaches for complete mobilization of the splenic flexure during laparoscopic rectal cancer resection. Int J Colorectal Dis. 27:1521-1529

Bhakta A, Tafen M, Glotzer O, Canete J, Chismark AD, Valerian BT, Stain SC, Lee EC (2016) Laparoscopic sigmoid colectomy for complicated diverticulitis is safe: review of 576 consecutive colectomies. Surg Endosc 30:1629-1634

Mino JS, Gandhi NS, Stocchi LL, Baker ME, Liu X, Remzi FH, Monteiro R, Vogel JD (2015) Preoperative risk factors and radiographic findings predictive of laparoscopic conversion to open procedures in Crohn's disease. J Gastrointest Surg 19:1007-1014

Kim NK, Kim YW, Han YD, Cho MS, Hur H, Min BS, Lee KY (2016) Complete mesocolic excision and central vascular ligation for colon cancer: Principle, anatomy, surgical technique, and outcomes. Surg Oncol 25:252-262

Merkel S, Weber K, Matzel KE, Agaimy A, Göhl J, Hohenberger W (2016) Prognosis of patients with colonic carcinoma before, during and after implementation of complete mesocolic excision. Br J Surg 103:1220-1229

Di Saverio S1 Vennix S, Birindelli A, Weber D, Lombardi R, Mandrioli M, Tarasconi A, Bemelman WA (2016) Pushing the envelope: laparoscopy and primary anastomosis are technically feasible in stable patients with Hinchey IV perforated acute diverticulitis and gross faeculent peritonitis. Surg Endosc. 30:5656-5664

Feinberg AE, Chesney TR, Acuna SA, Sammour T, Quereshy FA (2017) Oncologic Outcomes Following Laparoscopic versus Open Resection of pT4 Colon Cancer: A Systematic Review and Meta-analysis. Dis Colon Rectum 60:116-125

Vennix S, Lips DJ, Di Saverio S, van Wagensveld BA, Brokelman WJ, Gerhards MF, van Geloven AA, van Dieren S, Lange JF, 5.5 Bemelman WA (2016) Acute laparoscopic and open sigmoidectomy for perforated diverticulitis: a propensity score-matched $\mathbf{3 . 5}$ cohort

Surg Endosc 30:3889-3896 
Table 5 Seminal articles for flexible endoscopy pathway

5a: Diagnostic EGD/colonoscopy

Article Reference

CI

Google Scholar

Web of

Science

Lieberman BA, Rex DK, Winawer SJ, Giardiello FM, Johnson DA, Levin TR (2012) Guidelines for colonoscopy surveillance 185 after screening and polypectomy: a consensus update by the US Multi-Society Task Force on Colorectal Cancer. Gastroen- $\mathbf{1 2 2 . 8 3}$ terology 143:844-857

Rex DK, Petrini JL, Baron TH, Chak A, Cohen J, Deal SE, Hoffman B, Jacobson BC, Mergener K, Petersen BT, Safdi MA, 95.7

Faigel DO, Pike IM (2006) Quality indicators for colonoscopy. Gastrointest Endosc 63:S16-28

26.75

Barclay RL, Vicari JJ, Doughty AS, Johanson JF, Greenlaw RL (2006) Colonoscopic withdrawal times and adenoma detection 95.5 during screening colonoscopy. N Engl J Med 355:2533-2541

63.08

Winawer SJ, Zauber AG, Ho MN, O’Brien MJ, Gottlieb LS, Sternberg SS, Wayne JD, Schapiro M, Bond JH, Panish JF, et al. 182.36 (1993) Prevention of colorectal cancer by colonoscopic polypectomy. The National Polyp Study Workgroup. N Engl J Med 113.8 329:1977-1981

5b: Percutaneous endoscopic gastrostomy (PEG)

Article Reference

CI

Google Scholar

Web of Science

Larson DE, Burton DD, Schroeder KW, DiMango EP (1987) Percutaneous endoscopic gastrostomy. Indications, success, complications, and mortality in 314 consecutive patients. Gastroenterology 93:48-52

Wollman B, D’Agostino HB, Walus-Wigle JR, Easter DW, Beale A (1995) Radiologic, endoscopic, and surgical gastrostomy: an institutional evaluation and meta-analysis of the literature. Radiology 197:699-704

17.2

10.60

Kavic SM, Basson MD (2001) Complications of endoscopy. Am J Surg 181:319-332

12.1

6.05

5c: Endoscopic stent placement or dilation

Article Reference CI

Eubanks S, Edwards CA, Fearing NM, Ramaswamy A, de la Torre RA, Thaler KJ, Miedema BW, Scott JS (2008) Use of endoscopic stents to treat anastomotic complications after bariatric surgery. J Am Coll Surg 206:935-938

Swanstrom LL, Kurian A, Dunst CM, Sharata A, Bhayani N, Rieder E (2012) Long-term outcomes of an endoscopic myotomy 42.5 for achalasia: the POEM procedure. Ann Surg 256:659-667

27.0

Chan KC, Wong SK, Lee DW, Mui WL, Chan AC, Ng EK, Wu JC, Sung JJ, Chung SC (2004) Short-term and long-term 5.4 results of endoscopic balloon dilation for achalasia: 12 years' experience. Endoscopy 36:690-694

Table 6 Seminal articles for foregut pathway

6a: Laparoscopic Nissen fundoplication

Article Reference

CI

Google Scholar

Web of Science

Galmiche JP, Hatlebakk J, Attwood S, Ell C, Fiocca R, Eklund S, Långström G, Lind T, Lundell L; LOTUS Trial Collaborators (2011) Laparoscopic antireflux surgery vs esomeprazole treatment for chronic GERD: the LOTUS randomized clinical trial. JAMA 305:1969-1977

Spechler SJ, Lee E, Ahnen D, Goyal RK, Hirano I, Ramirez F, Raufman JP, Sampliner R, Schnell T, Sontag S, Vlahcevic ZR, Young R, Williford W (2001) Long-term outcome of medical and surgical therapies for gastroesophageal reflux disease: follow-up of a randomized controlled trial. JAMA 285:2331-2338

Rossi M, Barreca M, de Bortoli N, Renzi C, Santi S, Gennai A, Bellini M, Costa F, Conio M, Marchi S (2006) Efficacy of Nissen fundoplication versus medical therapy in the regression of low-grade dysplasia in patients with Barrett esophagus: a prospective study. Ann Surg 243:58-63

Oor JE, Roks DJ, Broeders JA, Hazebroek EJ, Gooszen HG (2017) Seventeen-year Outcome of a Randomized Clinical Trial Comparing Laparoscopic and Conventional Nissen Fundoplication: A Plea for Patient Counseling and Clarification. Ann Surg 266:23-28
45.6

25

60.8

34.11

7.9

3.75

8.0

2.0 
Table 6 (continued)

6a: Laparoscopic Nissen fundoplication

Article Reference

CI

Google Scholar

Web of Science

Broeders JA, Mauritz FA, Ahmed Ali U, Draaisma WA, Ruurda JP, Gooszen HG, Smout AJ, Broeders IA, Hazebroek EJ (2010) Systematic review and meta-analysis of laparoscopic Nissen (posterior total) versus Toupet (posterior partial) fundoplication for gastro-esophageal reflux disease. Br J Surg 97:1318-1330

Kinsey-Trotman SP, Devitt PG, Bright T, Thompson SK, Jamieson GG, Watson DI (2018) Randomized Trial of Division Ver- 1 sus Nondivision of Short Gastric Vessels During Nissen Fundoplication: 20-Year Outcomes. Ann Surg [Epub ahead of print]

Abdelrahman T, Latif A, Chan DS, Jones H, Farag M, Lewis WG, Havard T, Escofet X (2018) Outcomes after laparoscopic antireflux surgery related to obesity: A systematic review and meta-analysis. Int J Surg 51:76-82

Hunter JG, Kahrilas PJ, Bell RC, Wilson EB, Trad KS, Dolan JP, Perry KA, Oelschlager BK, Soper NJ, Snyder BE, Burch MA, Melvin WS, Reavis KM, Turgeon DG, Hungness ES, Diggs BS (2015) Efficacy of transoral fundoplication vs omeprazole for treatment of regurgitation in a randomized controlled trial. Gastroenterology 148:324-333.e5

Markar SR, Karthikesalingam AP, Hagen ME, Talamini M, Horgan S, Wagner OJ (2010) Robotic vs. laparoscopic Nissen fundoplication for gastro-esophageal reflux disease: systematic review and meta-analysis. Int J Med Robot 6:125-131

Jobe BA, Richter JE, Hoppo T, Peters JH, Bell R, Dengler WC, DeVault K, Fass R, Gyawali CP, Kahrilas PJ, Lacy BE, Pan19.6 dolfino JE, Patti MG, Swanstrom LL, Kurian AA, Vela MF, Vaezi M, DeMeester TR (2013) Preoperative diagnostic workup 8.0 before antireflux surgery: an evidence and experience-based consensus of the Esophageal Diagnostic Advisory Panel. J Am Coll Surg 217:586-597

6b: Laparoscopic paraesophageal hernia repair

Article Reference

CI

Google Scholar

Web of Science

Oelschlager BK, Pellegrini CA, Hunter J, Soper N, Brunt M, Sheppard B, Jobe B, Polissar N, Mitsumori L, Nelson J, 30.0

Swanstrom L (2006) Biologic prosthesis reduces recurrence after laparoscopic paraesophageal hernia repair: a multicenter, prospective, randomized trial. Ann Surg 244:481-490

Stadlhuber RJ, Sherif AE, Mittal SK, Fitzgibbons RJ Jr, Brunt LM, Hunter JG, Demeester TR, Swanstrom LL, Smith DC, Filipi CJ (2009) Mesh complications after prosthetic reinforcement of hiatal closure: a 28-case series. Surg Endosc 23:1219-1226

Frantzides CT, Madan AK, Carlson MA, Stavropolous GP (2002) A prospective, randomized trial of laparoscopic polytetrafluoroethylene (PTFE) patch repair vs simple cruroplasty for large hiatal hernia. Arch Surg 137:649-652

Stylopoulos N, Gazelle GS, Rattner DW (2002) Paraesophageal hernias: operation or observation? Ann Surg 236:492-500

Kohn GP, Price RR, DeMeester SR, Zehetner J, Muensterer OJ, Awad Z, Mittal SK, Richardson WS, Stefanidis D, Fanelli RD, 22.6

SAGES Guidelines Committee (2013) Guidelines for the management of hiatal hernia. Surg Endosc 27:4409-4428

\section{8}

Oelschlager BK, Petersen RP, Brunt LM, Soper NJ, Sheppard BC, Mitsumori L, Rohrmann C, Swanstrom LL, Pellegrini CA (2012) Laparoscopic paraesophageal hernia repair: defining long-term clinical and anatomic outcomes. J Gastrointest Surg $16: 453-459$

van der Westhuizen L, Dunphy KM, Knott B, Carbonell AM, Smith DE, Cobb WS 4th (2013) The need for fundoplication at the time of laparoscopic paraesophageal hernia repair. Am Surg 79:572-577

Hashemi M, Peters JH, DeMeester TR, Huprich JE, Quek M, Hagen JA, Crookes PF, Theisen J, DeMeester SR, Sillin LF, Bremner CG (2000) Laparoscopic repair of large type III hiatal hernia: objective follow-up reveals high recurrence rate. J Am Coll Surg 190:553-560

Jones R, Simorov A, Lomelin D, Tadaki C, Oleynikov D (2015) Long-term outcomes of radiologic recurrence after paraesophageal hernia repair with mesh. Surg Endosc 29:425-30

6c: Laparoscopic Heller myotomy

Article Reference

CI

Google Scholar

Web of Science

Patel DA, Lappas BM, Vaezi MF (2017) An Overview of Achalasia and Its Subtypes. Gastroenterol Hepatol (N.Y.) 13:4111.0 421

Kahrilas PJ, Pandolfino JE (2017) Treatments for achalasia in 2017: how to choose among them. Curr Opin Gastroenterol 33:270-276
10.8

6.0

2.2

0.8

25.1

14.5

4.7

3.0 
Table 6 (continued)

6c: Laparoscopic Heller myotomy

Article Reference

CI

Google Scholar

Web of Science

Nau P, Rattner D (2014) Laparoscopic Heller myotomy as the gold standard for treatment of achalasia. J Gastrointest Surg 18:2201-2207

Repici A, Fuccio L, Maselli R, Mazza F, Correale L, Mandolesi D, Bellisario C, Sethi A, Khashab MA, Rösch T, Hassan C (2018) GERD after peroral endoscopic myotomy as compared with Heller's myotomy with fundoplication: a systematic review with meta-analysis. Gastrointest Endosc 87:934-943.e18

Awaiz A, Yunus RM, Khan S, Memon B, Memon MA (2017) Systematic Review and Meta-Analysis of Perioperative Outcomes of Peroral Endoscopic Myotomy (POEM) and Laparoscopic Heller Myotomy (LHM) for Achalasia. Surg Laparosc Endosc Percutan Tech 27:123-131

Patti MG, Andolfi C, Bowers SP, Soper NJ (2017) POEM vs Laparoscopic Heller Myotomy and Fundoplication: Which Is Now the Gold Standard for Treatment of Achalasia? J Gastrointest Surg 21:207-214

Richter JE, Boeckxstaens GE (2011) Management of achalasia: surgery or pneumatic dilation. Gut 6:869-876

4.3

2.5

16.0

12.0

9.0

2.0

5.0

4.0

18.3

9.57

Smith CD, Stival A, Howell DL, Swafford V (2006) Endoscopic therapy for achalasia before Heller myotomy results in worse 17.1 outcomes than Heller myotomy alone. Ann Surg 243:579-584

9.16

Richards WO, Torquati A, Holzman MD, Khaitan L, Byrne D, Lufti R, Sharp KW (2004) Heller myotomy versus Heller 32.9 myotomy with Dor fundoplication for achalasia: a prospective randomized double-blind clinical trial. Ann Surg 240:405- 18.57 412

Rawlings A, Soper NJ, Oelschlager B, Swanstrom L, Matthews BD, Pellegrini C, Pierce RA, Pryor A, Martin V, Frisella 31.3 MM, Cassera M, Brunt LM (2012) Laparoscopic Dor versus Toupet fundoplication following Heller myotomy for achalasia: 18 results of a multicenter, prospective, randomized controlled trial. Surg Endosc 26:18-26

6d: Laparoscopic redo fundoplication

Article Reference

CI

Google Scholar

Web of Science

Furnée EJ, Draaisma WA, Broeders IA, Gooszen HG (2009) Surgical reintervention after failed antireflux surgery: a system- 14.2 atic review of the literature. J Gastrointest Surg 13:1539-1549

Smith CD, McClusky DA, Rajad MA, Lederman AB, Hunter JG (2005) When fundoplication fails: redo? Ann Surg 241:861869

11.1

5.84

Iqbal A, Awad Z, Simkins J, Shah R, Haider M, Salinas V, Turaga K, Karu A, Mittal SK, Filipi CJ (2006) Repair of 104 failed antireflux operations. Ann Surg 244:42-51

8.8

4.41

Khajanchee YS, O’Rourke R, Cassera MA, Gatta P, Hansen PD, Swanström LL (2007) Laparoscopic reintervention for failed 7.2 antireflux surgery: subjective and objective outcomes in 176 consecutive patients. Arch Surg 142:785-901

Dallemagne B, Arenas Sanchez M, Francart D, Perretta S, Weerts J, Markiewicz S, Jehaes C (2011) Long-term results after laparoscopic reoperation for failed antireflux procedures. Br J Surg 98:1581-1587

6.4

2.71

Lamb PJ, Myers JC, Jamieson GG, Thompson SK, Devitt PG, Watson DI (2009) Long-term outcomes of revisional surgery following laparoscopic fundoplication. Br J Surg 96:391-397

8.0

3.0

8.9

4.85 25:706-712

Byrne JP, Smithers BM, Nathanson LK, Martin I, Ong HS, Gotley DC (2005) Symptomatic and functional outcome after laparoscopic reoperation for failed antireflux surgery. Br J Surg 92:996-1001
5.1

2.69 
Table 7 Seminal articles for hernia pathway

7a: Laparoscopic primary ventral hernia repair

Article Reference

Google Scholar

Web of Science

Ventral Hernia Working Group, Breuing K, Butler CE, Ferzoco S, Franz M, Hultman CS, Kilbridge JF, Rosen M, Silverman

77.5

RP, Vargo D (2010) Incisional ventral hernias: review of the literature and recommendations regarding the grading and technique of repair. Surgery 148:544-558

Heniford BT, Park A, Ramshaw BJ, Voeller G (2003) Laparoscopic repair of ventral hernias: nine years' experience with 850 consecutive hernias. Ann Surg 238:391-399

Forbes SS, Eskicioglu C, McLeod RS, Okrainec A (2009) Meta-analysis of randomized controlled trials comparing open and laparoscopic ventral and incisional hernia repair with mesh. Br J Surg 96:851-858

Hiles M, Record Ritchie RD, Altizer AM (2009) Are biologic grafts effective for hernia repair?: a systematic review of the literature. Surg Innov 16:26-37

Burger JW, Halm JA, Wijsmuller AR, ten Raa S, Jeekel J (2006) Evaluation of new prosthetic meshes for ventral hernia repair. 21.4 Surg Endosc 20:1320-1325

Beldi G, Wagner M, Bruegger LE, Kurmann A, Candinas D (2011) Mesh shrinkage and pain in laparoscopic ventral hernia repair: a randomized clinical trial comparing suture versus tack mesh fixation. Surg Endosc 25:749-755

Tsereteli Z, Pryor BA, Heniford BT, Park A, Voeller G, Ramshaw BJ (2008) Laparoscopic ventral hernia repair (LVHR) in morbidly obese patients. 12:233-238

Deeken CR, Faucher KM, Matthews BD (2012) A review of the composition, characteristics, and effectiveness of barrier mesh 12.5 prostheses utilized for laparoscopic ventral hernia repair. Surg Endosc 26:566-575

Tandon A, Pathak S, Lyons NJ, Nunes QM, Daniels IR, Smart NJ (2016) Meta-analysis of closure of the fascial defect during laparoscopic incisional and ventral hernia repair. Br J Surg 103:1598-1607

Sauerland S, Walgenbach M, Habermalz B, Seiler CM, Miserez M (2011) Laparoscopic versus open surgical techniques for

7b: Laparoscopic primary inguinal hernia repair

Article Reference

CI

Google Scholar

Web of Science

McCormack K, Scott NW, Go PM, Ross S, Grant AM, EU Hernia Trialists Collaboration (2003) Laparoscopic techniques versus open techniques for inguinal hernia repair. Cochrane Database Syst Rev 1:CD001785

Neumayer L, Giobbie-Hurder A, Jonasson O, Fitzgibbons R Jr, Dunlop D, Gibbs J, Reda D, Henderson W, Veterans Affairs Cooperative Studies Program 456 Investigators (2004) Open mesh versus laparoscopic mesh repair of inguinal hernia. N Engl J Med 350:1819-1827

Klinge U, Klosterhalfen B, Müller M, Schumpelick V (1999) Foreign body reaction to meshes used for the repair of abdominal 21.1 wall hernias. Eur J Surg 165:665-673

Liem MS, van Duyn EB, van der Graaf Y, van Vroonhoven TJ; Coala Trial Group (2003) Recurrences after conventional anterior and laparoscopic inguinal hernia repair: a randomized comparison. Ann Surg 237:136-141

Katkhouda N, Mavor E, Friedlander MH, Mason RJ, Kiyabu M, Grant SW, Achanta K, Kirkman EL, Narayanan K, Essani R (2001) Use of fibrin sealant for prosthetic mesh fixation in lap extraperitoneal inguinal hernia repair. Ann Surg 233:18-25

Li J, Ji Z, Li Y (2014) Comparison of laparoscopic versus open procedure in the treatment of recurrent inguinal hernia: a meta-analysis of the results. Am J Surg 207:602-612

Deeken CR, Abdo MS, Frisella MM, Matthews BD (2011) Physicomechanical evaluation of polypropylene, polyester, and polytetrafluoroethylene meshes for inguinal hernia repair. J Am Coll Surg 212:68-79

HerniaSurge Group (2018) International guidelines for groin hernia management. Hernia 22:1-165

Antoniou SA, Köhler G, Antoniou GA, Muysoms FE, Pointner R, Granderath FA (2016) Meta-analysis of randomized trials comparing nonpenetrating vs mechanical mesh fixation in laparoscopic inguinal hernia repair. Am J Surg 211:239-249.e2

Sajid MS, Kalra L, Parampalli U, Sains PS, Baig MK (2013) A systematic review and meta-analysis evaluating the effectiveness of lightweight mesh against heavyweight mesh in influencing the incidence of chronic groin pain following laparoscopic $\mathbf{4 . 4}$ inguinal hernia repair. Am J Surg 205:726-736
57.9

0.1

75.4

35.9

13.1

13.8

5.7

11.5 
Table 7 (continued)

7c: Laparoscopic redo hernia repairs or atypical location hernia repairs

Article Reference

CI

Google Scholar

Web of Science

Zhou DJ, Carlson MA (2018) Incidence, etiology, management, and outcomes of flank hernia: review of published data.

Hernia 22:353-361

1

Hope WW, Hooks WB 3rd (2013) Atypical hernias: suprapubic, subxiphoid, and flank. Surg Clin North Am 93:1135-1162 3.8

Sharma A, Khullar R, Soni V, Baijal M, Kapahi A, Najma K, Chowbey PK (2013) Iatrogenic enterotomy in laparoscopic 4.4

ventral/incisional hernia repair: a single center experience of 2,346 patients over 17 years. Hernia 17:581-587

Renard Y, Simonneau AC, de Mestier L, Teuma L, Meffert JL, Palot JP, Kianmanesh R (2017) Standard of Open Surgical 2.0

Repair of Suprapubic Incisional Hernias. World J Surg 41:1466-1474

DeAsis FJ, Lapin B, Gitelis ME, Ujiki MB (2015) Current state of laparoscopic parastomal hernia repair: A meta-analysis. 14.0

World J Gastroenterol 21:8670-8677

Jones HG, Rees M, Aboumarzouk OM, Brown J, Cragg J, Billings P, Carter B, Chandran P (2018) Prosthetic mesh place- 4 ment for the prevention of parastomal herniation. Cochrane Database Syst Rev 7:CD008905

Pisanu A, Podda M, Saba A, Porceddu G, Uccheddu A (2015) Meta-analysis and review of prospective randomized trials 17.0

comparing laparoscopic and Lichtenstein techniques in recurrent inguinal hernia repair. Hernia 19:355-366

Cornette B, De Bacquer D, Berrevoet F (2018) Component separation technique for giant incisional hernia: A systematic 10

review. Am J Surg 215:719-726

Warren JA, McGrath SP, Hale AL, Ewing JA, Carbonell AM 2nd, Cobb WS 4th (2017) Patterns of Recurrence and 5.0

Mechanisms of Failure after Open Ventral Hernia Repair with Mesh. Am Surg 83:1275-1282 3.0

Patel PP, Love MW, Ewing JA, Warren JA, Cobb WS, Carbonell AM (2017) Risks of subsequent abdominal operations 11.0

after laparoscopic ventral hernia repair. Surg Endosc 31:823-828

.8
4
.0
.0
.0
4.0
.3

7.0
.0
0
4.0
3.0
1.0
3.0

Table 8 Seminal articles for robotic surgery pathway

Article Reference

CI

Google Scholar

Web of Science

Vilallonga R, Fort JM, Caubet E, Gonzalez O, Armengol M (2013) Robotic sleeve gastrectomy versus laparoscopic sleeve

gastrectomy: a comparative study with 200 patients. Obes Surg 23:1501-1507

4.2

Strosberg DS, Nguyen MC, Muscarella P 2nd, Narula VK (2017) A retrospective comparison of robotic cholecystectomy versus laparoscopic cholecystectomy: operative outcomes and cost analysis. Surg Endosc 31:1436-1441

Carbonell AM, Warren JA, Prabhu AS, Ballecer CD, Janczyk RJ, Herrera J, Huang LC, Phillips S, Rosen MJ, Poulose BK

(2018) Reducing Length of Stay Using a Robotic-assisted Approach for Retromuscular Ventral HerniaRepair: A Comparative Analysis From the Americas Hernia Society Quality Collaborative. Ann Surg 267:210-217

Galvani CA, Loebl H, Osuchukwu O, Samamé J, Apel ME, Ghaderi I (2016) Robotic-Assisted Paraesophageal Hernia Repair: Initial Experience at a Single Institution. J Laparoendosc Adv Surg Tech A 26:290-295

Jayne D, Pigazzi A, Marshall H, Croft J, Corrigan N, Copeland J, Quirke P, West N, Rautio T, Thomassen N, Tilney H, Gudg- 69.5 eon M, Bianchi PP, Edlin R, Hulme C, Brown J (2017) Effect of Robotic-Assisted vs Conventional Laparoscopic Surgery on 42.5 Risk of Conversion to Open Laparotomy Among Patients Undergoing Resection for Rectal Cancer: The ROLARR Randomized Clinical Trial. JAMA 318:1569-1580

Warren JA, Cobb WS, Ewing JA, Carbonell AM (2017) Standard laparoscopic versus robotic retromuscular ventral hernia repair. Surg Endosc 31:324-332

Brody F, Richards NG (2014) Review of robotic versus conventional laparoscopic surgery. Surg Endosc 28:1413-1424

Shaligram A, Unnirevi J, Simorov A, Kothari VM, Oleynikov D (2012) How does the robot affect outcomes? A retrospective 10.9 review of open, laparoscopic, and robotic Heller myotomy for achalasia. Surg Endosc 26:1047-1050

Toro JP, Lin E, Patel AD (2015) Review of robotics in foregut and bariatric surgery. Surg Endosc 29:1-8 5.5 


\section{Discussion}

In this paper, using a systematic methodology and expert consensus, we identified and reported the seminal articles for each anchoring procedure of the eight SAGES Masters pathways. The goal of this project was to provide participants of the SAGES Masters pathways with the best available literature relevant for each procedure. The steering group of the Masters program felt that these papers would be invaluable for surgeon participants of the program to ensure best possible acquisition of knowledge relevant to each procedure. Identifying articles that have exerted the most influence on a particular procedure and field could help surgeons become familiar with landmark works, recognize optimal procedural outcomes, better understand the mechanisms of action, and identify aspects of each procedure and technique that deserve more attention. Further, by identifying the top published articles, insight is provided into knowledge generation processes that may be vital to surgical education and research; these articles may highlight the types of work that have the most impact in a field and become "classics". This process also honors leaders in the field by identifying the impact of their work [3].

Other studies have identified the top-cited articles in urology, [4] orthopedic surgery [5], arthroscopy, [6] emergency medicine, [7] plastic surgery, [8] and medical education [3]. Nevertheless, we could not identify publications relevant to seminal papers in general surgery and the anchoring procedures of the SAGES Masters program, which is why we undertook this project.

Similar to our work, prior studies have used citation frequency to identify the top articles in their field [3-8]. A distinct difference of our study's methodology, however, is that we subjected the initially identified articles based on citation frequency to expert review and feedback. The latter determined the final list of sentinel articles. Our rationale was that for the purposes of this project, identifying the seminal articles for each procedure was a more appropriate approach as our focus was on teaching rather than the research impact of the top articles. While we took into consideration the impact of each article based on number of citations, our ultimate goal was to provide surgeons with the most relevant articles to each procedure with regard to technical considerations, pathophysiology, effectiveness, and patient outcomes.

Unlike prior studies, we also used a citation index instead of solely the number of citations. Our justification was that articles published earlier have more time to accumulate citations compared to more recent literature. While our approach is based on a solid rationale, the impact of time on citation number has not been proven [7]. It should also be noted that we searched the Web of Science, while other relevant papers have used the Web of Knowledge, which is a more comprehensive database of the literature. Nevertheless, Web of Science includes all relevant surgical journals and the additional expert review we conducted should have minimized potentially missing articles. Unlike prior publications that have limited their search to specific surgical journals, [5, 9] our approach provided a broader search of the literature and included all journals indexed in Web of Science.

Known limitations of identifying "top" articles [5, 10, 11] based on citation analysis include not accounting for self-citations, citations in textbooks, article popularity, and electronic views of an article, and authors' potential preference to cite articles in the journal in which they seek to publish their work $[5,12]$. The expert review we conducted may have addressed some of these limitations. On the other hand, expert reviews introduce their own biases such as selecting only articles they are familiar with or only consider those that reflect own experiences and biases. We believe we have minimized these biases by including both large number of experts and relying on consensus as well as by combining citation numbers with expert opinion. Given that the literature continues to accumulate and evolve, it is also important to note, that the process we followed in this study will need to be repeated in due time in the future (3-5 years) in order to ensure surgeons learn using the most up-to-date references.

In summary, we have identified the seminal articles for all anchoring procedures of the SAGES Masters program pathways using a systematic methodology. We believe that these articles will add value to the Masters pathways by providing surgeon participants a great resource to improve their procedural knowledge. They may further benefit the larger surgical community by focusing its attention to must-read impactful work that may inform best practices.

Acknowledgements We want to thank the following SAGES members who helped select the top 10 papers for each masters pathway: Deborah Keller, MS, MD, Sami Chadi, MD, MSc, Lawrence Lee, MD, PhD, Tonia Young-Fadock, MD, MS, Brian R. Davis, MD, B. Fernando Santos, MD, Sunny Shah MD, Saniea Majid MD, Matt Kroh MD, Nabeel Obeid MD, Vamsi Alli, MD, Antonio Caycedo, MD, Ryan Juza, MD, David Urbach, MD, Mazen Al-Mansour, MD, Abdulrahim Al Awashez, MD, Salvatore Docimo, MD, Konstantinos Makris, MD, Jim Ellsemere, MD, Emmanuele Lo Menzo, MD, Patrick Reardon, MD, Ankit Patel, MD, Michael Truitt, MD, Samer Sbayi, MD, Jonathan Pearl, MD, Nawar Alkhamesi, MD, Josh Weis, MD, Ben Clark, MD, Matt Bloom, MD, Dan Bonville, MD, Jon Roth, MD, Melissa Hogg, MD, Seth Rosen, MD, Seth Felder, MD, JB Bittner, MD, Yusef Kudsi, MD, Conrad Ballecer, MD, Alberto Meyer, MD, Ambar Banerjee, MD, Andrea Pakula, MD, Archana Ramaswamy, MD, Caitlyn Lesh, MD, David Earle, MD, Fernando Garcia, MD, Gabriel Arevalo, MD, Hussna Wakily, MD, Jake Greenberg, MD, Jeff Blatnik, MD, Julietta Chang, MD, Rebecca Peterson, MD, Rich Pierce, MD, Stephen Haggerty, MD, and Stephen McNatt, MD.

Funding This project did not receive any funding. 


\section{Compliance with ethical standards}

Disclosures Dr. Patricia Sylla reports grants and personal fees from Karl Storz, grants and personal fees from Olympus, grants and personal fees from Ethicon, grants and personal fees from Medtronic, outside the submitted work. Dr. Eric M. Pauli reports personal fees from Boston Scientific, personal fees from Cook Biotech, grants and personal fees from CR Bard, outside the submitted work.kimca. Dr. Dmitry Oleynikov reports personal fees and other financial ties from Virtual Incision, grants and nonfinancial support from Gore, outside the submitted work. Dr. Marina Kurian reports personal fees from Gore, personal fees from Ethicon, personal fees from Allurion, personal fees from Medtronic, outside the submitted work. Dr. Leena Khaitan has no conflicts of interest or financial ties to disclose. Dr. Michael W. Cripps reports personal fees from Hemosonics, personal fees from Instrumentation Laboratory Worldwide, outside the submitted work. Dr. Sharon Bachman has no conflicts of interest or financial ties to disclose. Dr. Adnan Alseidi has no conflicts of interest or financnial ties to disclose. Dr. L. Michael Brunt reports grants from Gore Medical, personal fees from Intutive Surgical, outside the submitted work. Dr. Horacio Asbun reports other financial ties from Boston Scientific, other financial ties from Olympus, outside the submitted work. Dr. Daniel B. Jones reports other financial ties from Allurion, outside the submitted work. Dr. Dimitrios Stefanidis, Linda Schultz and Shauna Bostian have no conflict of interest or financial ties to disclose

\section{References}

1. Jones DB, Stefanidis D, Korndorffer JR Jr, Dimick JB, Jacob BP, Schultz L, Scott DJ (2017) SAGES University MASTERS Program: a structured curriculum for deliberate, lifelong learning. Surg Endosc 31:3061-3071
2. Dreyfus SE, Dreyfus HL (February 1980) A five-stage model of the mental activities involved in directed skill acquisition. DTIC ADA084551. University of California/Berkeley Operations Research Center

3. Azer SA (2015) The top-cited articles in medical education: a bibliometric analysis. Acad Med 90:1147-1161

4. Heldwein FL, Rhoden EL, Morgentaler A (2010) Classics of urology: a half century history of the most frequently cited articles (1955-2009). Urology 75:1261-1268

5. Lefaivre KA, Shadgan B, O'Brien PJ (2011) 100 most cited articles in orthopaedic surgery. Clin Orthop Relat Res 469:1487-1497

6. Cassar Gheiti AJ, Downey RE, Byrne DP, Molony DC, Mulhall $\mathrm{KJ}$ (2012) The 25 most cited articles in arthroscopic orthopaedic surgery. Arthroscopy 28:548-564

7. Munzer BW, Love J, Shipman BL, Byrne B, Cico SJ, Furlong R, Khandelwal S, Santen SA (2017) An analysis of the top-cited articles in emergency medicine education literature. West J Emerg Med 18:60-68

8. Joyce CW, Carroll SM (2014) Microsurgery: the top 50 classic papers in plastic surgery: a citation analysis. Arch Plast Surg 41:153-157

9. Kelly JC, Glynn RW, O’Briain DE, Felle P, McCabe JP (2010) The 100 classic papers of orthopaedic surgery: a bibliometric analysis. J Bone Joint Surg Br 92:1338-1343

10. Garfield E, Cawkell AE (1975) Citation analysis studies. Science 189(4200):397. https://doi.org/10.1126/science.189.4200.397

11. Callaham M, Wears RL, Weber R (2002) Journal prestige, publication bias, and other characteristics associated with citation of published studies in peer-reviewed journals. JAMA 287:2847-2850

12. Seglen PO (1997) Citations and journal impact factors: Questionable indicators of research quality. Allergy 52:1050-1056

Publisher's Note Springer Nature remains neutral with regard to jurisdictional claims in published maps and institutional affiliations. 